\title{
Resuscitative Endovascular Balloon Occlusion of the Aorta for an Iliac Artery Aneurysm: Case Report
}

\author{
Sung Wook Chang, M.D. ${ }^{1,2}$, Sangwook Chun, M.D. ${ }^{2}$, Gyeongho Lee, M.D. ${ }^{2}$, Pil Won Seo, M.D., Ph.D. ${ }^{2}$ \\ ${ }^{1}$ Trauma Center and ${ }^{2}$ Department of Thoracic and Cardiovascular Surgery, Dankook University Hospital, Cheonan, Korea
}

\section{ARTICLE INFO}

Received September 25, 2020

Accepted October 22, 2020

Corresponding author

Pil Won Seo

Tel 82-41-550-6195

Fax 82-41-550-0039

E-mail pwseo@dankook.ac.kr ORCID

https://orcid.org/0000-0003-0084-1814

\begin{abstract}
Isolated iliac artery aneurysm (IAA) is rare, but can be fatal. Emergency surgery is performed in cases of hemorrhagic shock due to a suddenly ruptured IAA, which may have a high mortality rate because of massive non-compressible torso hemorrhage (NCTH). Recently, resuscitative endovascular balloon occlusion of the aorta (REBOA) has been accepted as an alternative to aortic cross-clamping via open thoracotomy to achieve hemostasis in trauma patients with profound shock due to NCTH and is considered an emerging bridging therapy for damage control. However, there is limited information on the use of REBOA in non-trauma patients with shock. Herein, we describe a patient with impending cardiac arrest due to isolated ruptured IAA, in whom perioperative bleeding was successfully controlled by REBOA.
\end{abstract}

Keywords: Iliac artery, Aneurysm, Rupture, Aorta, Balloon occlusion, Case report

\section{Case report}

A 67-year-old woman with hypertension presented to the emergency department with a chief complaint of abdominal pain, which had started 6 hours prior. On arrival, her vital signs were as follows: blood pressure (BP), 156/66 mm $\mathrm{Hg}$; pulse rate, 76 beats/min; respiratory rate, 17 breaths/ min; oxygen saturation, $100 \%$; and body temperature, $36.6^{\circ} \mathrm{C}$. Physical examination revealed a mass-like lesion with tenderness in the right lower quadrant of the abdomen. Contrast-enhanced computed tomography (CT) of the abdominopelvic region revealed a ruptured external iliac artery (EIA) aneurysm with extensive active extravasation and a huge retroperitoneal hematoma (Fig. 1A, B). After the CT scan, the patient's systolic blood pressure (SBP) decreased to $40 \mathrm{~mm} \mathrm{Hg}$. Despite fluid resuscitation and vasopressor support, her SBP reduced further to $30 \mathrm{~mm}$ $\mathrm{Hg}$. We decided to perform chest compressions simultaneously with resuscitative endovascular balloon occlusion of the aorta (REBOA) for damage control in an attempt to mitigate impending cardiac arrest.

Ultrasound-guided vascular access to the left common
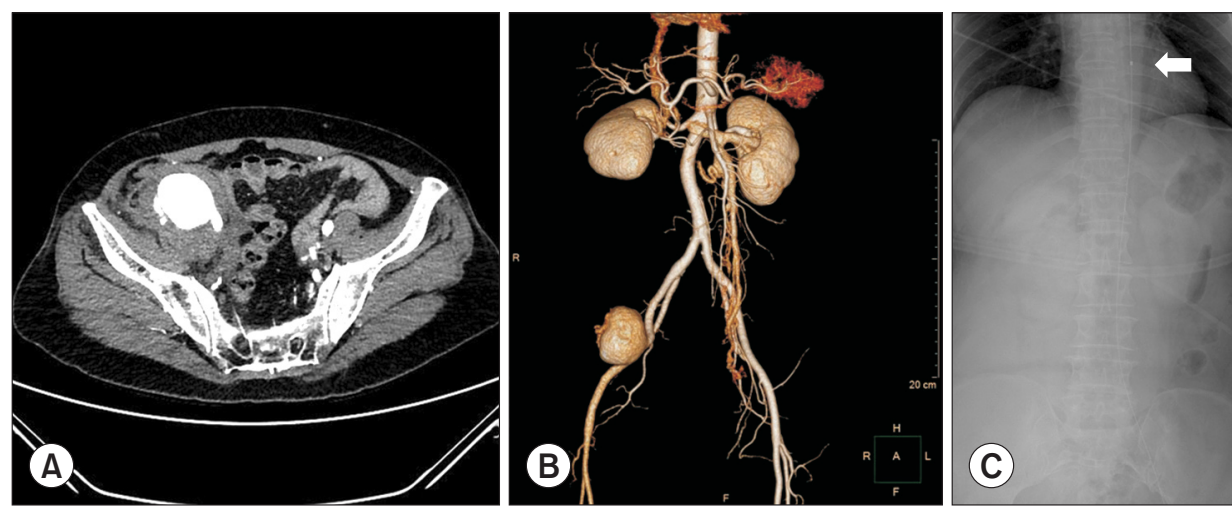

Fig. 1. Computed tomography (CT) scan and radiograph. (A) CT scan showing the site of active bleeding from the external iliac artery and a huge hematoma. (B) CT vascular reconstruction showing the isolated iliac artery aneurysm with extravasation. (C) Radiograph showing zone I resuscitative endovascular balloon occlusion of the aorta with the balloon located above the diaphragm (white arrow: the bottom of the balloon catheter with the radiopaque steel marker).

Copyright $($ C) 2021, The Korean Society for Thoracic and Cardiovascular Surgery

(c) (i) \& This is an Open Access article distributed under the terms of the Creative Commons Attribution Non-Commercial License (http://creativecommons.org/licenses/ by-nc/4.0) which permits unrestricted non-commercial use, distribution, and reproduction in any medium, provided the original work is properly cited. 
Table 1. Timing of critical events in the patient's course

\begin{tabular}{|c|c|c|c|}
\hline Time & $\begin{array}{l}\text { Blood pressure } \\
\quad(\mathrm{mm} \mathrm{Hg})\end{array}$ & Events & Additional notes \\
\hline 05:00 & & Abdominal pain & \\
\hline $11: 11$ & $156 / 66$ & Presented to emergency department & \\
\hline $11: 53$ & $160 / 98$ & Sent to CT room & \\
\hline $12: 20$ & $40 / 32$ & $\begin{array}{l}\text { Returned from CT room; aggravation of } \\
\text { abdominal pain }\end{array}$ & $\begin{array}{l}\text { Intubated; fluid resuscitation; right subclavian vein line } \\
\text { placed; norepinephrine infusion initiated }\end{array}$ \\
\hline $12: 37$ & $30 / 24$ & Chest compression & Prepared to perform REBOA; 3 units of PRBCs transfused \\
\hline $12: 44$ & $24 / 17$ & $7 F$ sheath insertion & Ultrasound-guided cannulation \\
\hline $12: 46$ & $32 / 17$ & REBOA balloon insertion; zone I REBOA & X-ray examination; inflated to $13 \mathrm{~mL}$, partial occlusion \\
\hline $12: 57$ & $101 / 70$ & Changed to zone III REBOA & $25 \mathrm{~cm}$ withdrawal; $11 \mathrm{~min}$ for zone I REBOA \\
\hline 13:00 & $93 / 70$ & Transferred to operating room & \\
\hline $13: 30$ & & $\begin{array}{l}\text { EIA exposure; proximal and distal EIA occlusion } \\
\text { via arteriotomy }\end{array}$ & Norepinephrine infusion off; use of Fogarty catheter \\
\hline $13: 34$ & & Full deflation of REBOA balloon & 37 min for zone III REBOA \\
\hline $14: 35$ & & Removal of REBOA balloon & Total occlusion time: $48 \mathrm{~min}$ \\
\hline
\end{tabular}

CT, computed tomography; REBOA, resuscitative endovascular balloon occlusion of the aorta; PRBC, packed red blood cell; EIA, external iliac artery.

femoral artery was used to place a 7F Radiofocus Introducer II (Terumo, Tokyo, Japan) sheath. A 7F RESCUE Balloon (Tokai Medical Products, Kasugai, Japan) was deployed using the xyphoid process as an external anatomical landmark via the sheath. The length of the inserted balloon was $50 \mathrm{~cm}$. Noting no resistance, the balloon was initially inflated to $13 \mathrm{~mL}$ for partial occlusion of the descending thoracic aorta. After balloon occlusion, the patient's SBP improved from 30 to $95 \mathrm{~mm} \mathrm{Hg}$. The ideal location for zone I REBOA was then confirmed radiographically (Fig. 1C). Following transfusion with 3 units of packed red blood cells, SBP was maintained at $100 \mathrm{~mm} \mathrm{Hg}$. To minimize ischemic complications, the balloon catheter was moved $25 \mathrm{~cm}$ caudally to change to zone III REBOA with no findings of hemodynamic deterioration. The patient was then transferred to the operating room (Table 1).

A retroperitoneal approach via a Rutherford Morrison incision revealed a huge hematoma, $4 \mathrm{~cm}$ in diameter, and a ruptured EIA aneurysm with active bleeding (Fig. 2). Extended arteriotomy of the rupture site and intra-arterial balloon occlusion were performed at the proximal and distal EIA using 5F Fogarty occlusion catheters (Edwards, Irvine, CA, USA). Next, the REBOA balloon was slowly deflated under closed monitoring. Aneurysmectomy and angioplasty with bovine pericardium were then completed. The total time period of partial aortic occlusion was 48 minutes (11 minutes in zone I REBOA and 37 minutes in zone III REBOA), and the REBOA balloon catheter was removed in the operating room. Her vital signs stabilized postoperatively, and she was discharged without any complaints 24 days postoperatively.

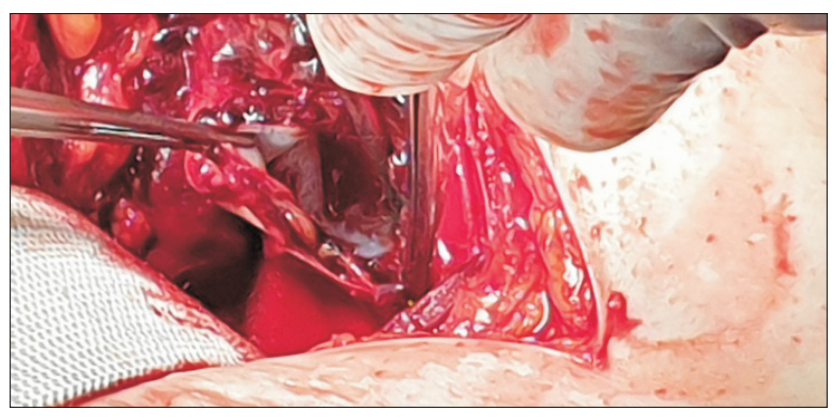

Fig. 2. Intraoperative findings show a ruptured external iliac artery aneurysm. No extensive bleeding from the proximal iliac artery occurred due to partial occlusion by zone III resuscitative endovascular balloon occlusion of the aorta.

The study was approved by the Institutional Review Board of Dankook University Hospital (IRB approval no., 2020-10-023). The requirement for informed consent was waived.

\section{Discussion}

Iliac artery aneurysms (IAAs) are uncommon and frequently occur in conjunction with abdominal aortic aneurysms [1,2]. Isolated IAAs are rare, accounting for fewer than $1.9 \%$ of all intra-abdominal aneurysms [1,2]. As IAAs are often masked by nonspecific signs and symptoms, early diagnosis and treatment may be difficult. Surgical repair is considered to be the gold standard. Although the mortality rate has been reported to be low in the elective management of unruptured IAAs, ruptured IAAs are associated with significant operative mortality of up to $50 \%$ [3]. With 
advances in endovascular technology and techniques, percutaneous endovascular stent-graft repair of IAA has the potential to reduce perioperative mortality [4]. However, if endovascular management is not available immediately due to a lack of an appropriate stent graft or if there is no time to prepare for surgery or intervention for patients with impending cardiac arrest, damage control resuscitation should be performed regardless of the patient's location.

REBOA has been accepted as a technique for preoperatively reducing hemorrhage and increasing cerebral/coronary perfusion in patients with non-compressible torso hemorrhage (NCTH) [5-7]. Recently, its use has expanded beyond just trauma care to encompass other types of bleeding or resuscitation for hemodynamic stabilization [5-8]. In general, different occlusion zones in the aorta could be used to block blood flow to the lower body with the following classification and indications: zone I REBOA (descending thoracic aorta, from the origin of the left subclavian artery to the celiac artery) for patients with hemoperitoneum and/or retroperitoneal hemorrhage and zone III REBOA (infrarenal abdominal aorta, from the renal artery to the aortic bifurcation) for patients with pelvic bleeding. Several reports have suggested that zone I REBOA may more optimally elevate proximal BP than zone III REBOA in patients with hemorrhagic shock $[9,10]$. Therefore, zone I REBOA should be preferentially considered in patients with profound shock regardless of the bleeding focus $[9,10]$. In this case, despite massive bleeding from a ruptured IAA, zone I REBOA was performed for damage control because the patient's status rapidly deteriorated and resulted in impending cardiac arrest.

Perfusion-related complications following REBOA, such as acute kidney injury and leg ischemia, have been reported $[7,9,10]$. Therefore, it is important to perform safeguards to minimize ischemic complications below the site of the occlusion. One safeguard is to find the optimal occlusion level and another is to choose the proper occlusion method. In this case, zone I REBOA was performed to elevate the SBP initially. After stabilizing the patient's hemodynamics, the REBOA balloon catheter was moved to a zone III occlusion in order to maintain adequate renal and bowel perfusion. Initial CT showed that the aortic bifurcation was located along the upper margin of the fourth lumbar vertebra. Post-insertion radiography showed that the distance from the bottom of the balloon catheter, with a radiopaque steel marker, to the fourth lumbar vertebra was 25 $\mathrm{cm}$. Thus, the balloon was withdrawn $25 \mathrm{~cm}$ in the caudal direction. Another important step taken in this case was the use of permissive hypotension as a resuscitative strategy. The balloon was initially inflated to $13 \mathrm{~mL}$ to occlude the aorta partially, without any resistance, and the distal pulse of the dorsalis pedis artery was palpated after stabilizing the patient's hemodynamics. We believe that the patient did not experience any perfusion-related complications because these principles were followed.

In conclusion, ruptured isolated IAA is rare, but is associated with a significant risk of operative mortality. If a ruptured IAA with massive hemorrhage is diagnosed, immediate treatment such as surgery or a vascular intervention is essential to reduce mortality. In this case, in the setting of impending cardiac arrest due to ruptured IAA, REBOA was successfully performed as a damage control procedure. Therefore, REBOA could be a useful option to allow adequate time to prepare for surgery and to stabilize hemodynamics in the interim. Timely use of REBOA in cases of non-traumatic IAA rupture could reduce the perioperative mortality rate and may be a life-saving procedure.

\section{Conflict of interest}

No potential conflict of interest relevant to this article was reported.

\section{ORCID}

Sung Wook Chang: https://orcid.org/0000-0002-2689-3068 Sangwook Chun: https://orcid.org/0000-0003-1561-442X Gyeongho Lee: https://orcid.org/0000-0001-5928-3939

Pil Won Seo: https://orcid.org/0000-0003-0084-1814

\section{References}

1. Chaer RA, Barbato JE, Lin SC, Zenati M, Kent KC, McKinsey JF. Isolated iliac artery aneurysms: a contemporary comparison of endovascular and open repair. J Vasc Surg 2008;47:708-13.

2. Kimura F, Ookubo R, Kobayashi D, Harada H, Baba T. Successful endovascular repair of a ruptured isolated iliac artery aneurysm: a case report. Clin Case Rep 2019;7:1880-4.

3. Richardson JW, Greenfield LJ. Natural history and management of iliac aneurysms. J Vasc Surg 1988;8:165-71.

4. Boules TN, Selzer F, Stanziale SF, et al. Endovascular management of isolated iliac artery aneurysms. J Vasc Surg 2006;44:29-37.

5. Cho S, Ryu JW, Chang SW. Resuscitative endovascular balloon occlusion of the aorta for retroperitoneal hemorrhage and shock after ipsilateral antegrade angioplasty with vascular closure device. Ann Transl Med 2020;8:404. 
6. Horer T. Resuscitative endovascular balloon occlusion of the aorta (REBOA) and endovascular resuscitation and trauma management (EVTM): a paradigm shift regarding hemodynamic instability. Eur J Trauma Emerg Surg 2018;44:487-9.

7. Kim DH, Chang SW, Matsumoto J. The utilization of resuscitative endovascular balloon occlusion of the aorta: preparation, technique, and the implementation of a novel approach to stabilizing hemorrhage. J Thorac Dis 2018;10:5550-9.

8. Chang SW, Chang YR, Seon YJ, Park JW, Kim JS. Intraoperative use of resuscitative endovascular balloon occlusion of the aorta for hem- orrhage control in woman with placenta percreta involving the bladder. Perinatology 2019;30:175-8.

9. Beyer CA, Johnson MA, Galante JM, DuBose JJ. Zones matter: hemodynamic effects of zone 1 vs zone 3 resuscitative endovascular balloon occlusion of the aorta placement in trauma patients. Injury 2019;50:855-8.

10. Tibbits EM, Hoareau GL, Simon MA, et al. Location is everything: the hemodynamic effects of REBOA in zone 1 versus zone 3 of the aorta. J Trauma Acute Care Surg 2018;85:101-7. 